\title{
Comparison of Localization Free Routing Protocols in Underwater Wireless Sensor Networks
}

\author{
Muhammad Khalid \\ Institute of Management Sciences \\ Peshawar Pakistan, 25000
}

\author{
Awais Adnan \\ Institute of Management Sciences \\ Peshawar Pakistan, 25000
}

\author{
Zahid Ullah \\ Institute of Management Sciences \\ Peshawar Pakistan, 25000
}

Waqar Khalid

Institute of Management Sciences

Peshawar Pakistan, 25000

\author{
Naveed Ahmad \\ Department of Computer Science \\ University of Peshawar, Pakistan 25000
}

\author{
Ahsan Ashfaq \\ Institute of Management Sciences \\ Peshawar Pakistan, 25000
}

\begin{abstract}
Underwater Wireless Sensor Network (UWSN) is newly developed branch of Wireless Sensor network (WSN). UWSN is used for exploration of underwater resources, oceanographic data collection, flood or disaster prevention, tactical surveillance system and unmanned underwater vehicles. UWSN uses sensors of small size with a limited energy, memory and allows limited range for communication. Due to multiple differences from terrestrial sensor network, radio waves cannot be used over here. Acoustic channel are used for communication in deep water, which has many limitations like low bandwidth, high end to end delay and path loss. With the above limitations while using acoustic waves, it is very important to develop energy efficient and reliable protocols. Energy efficient communication in underwater networks has become uttermost need of UWSN technology. The main aim nowadays is to operate sensor with smaller battery for a longer time. This paper will analyse various routing protocols in the area of UWSN through simulation. This paper will analyse Depth Based Routing (DBR), Energy Efficient Depth Based Routing (EEDBR) and Hop by Hop Dynamic Addressing Based (H2-DAB) protocol through simulation. This comparison is carried out on the basis of total consumed energy, end to end delay, path loss and data delivery ratio.
\end{abstract}

Keywords-Underwater Networks; Sensor; Wireless Communication; Survey; Localization Based; Routing; Protocols

\section{INTRODUCTION}

UWSN is a newly emerging wireless technology which is providing the most promising mechanism used for discovering acoustic environment very efficiently for many scenarios like military [1], emergency and commercial purposes [2]. Autonomous Underwater and unmanned Vehicles which are equipped with sensors that are specially designed for underwater communication, which are mostly used in those areas where exploration for natural resources which lies underwater is needed. These unmanned vehicle gather those data and send it back to off shore sinks which is forwarded to other stations for further processing[3]. Radio waves cannot be used in underwater communication. Therefore, communication is made through acoustic channels. Once data packet reaches sink then it is forwarded through radio waves to other sinks and stations [4]. Underwater wireless sensor environment is much different from that of terrestrial network where no such ambiguities are found which we face in underwater communication while using radio communication [5]. Normally the problems we faces during communication in underwater communication are dense salty water, electromagnetic as well as optical signal does not work here [6]. Due to high attenuation and absorption effect, signals cannot travel long distances. Hence to overcome these problems acoustic communication, is used[7]. It can overcome these problem and provides a better transfer rate in underwater environment[8]. Using acoustic communication propagation speed lowered down from speed of light to that of sound speed which is $1500 \mathrm{~m} / \mathrm{sec}$. Due to lower speed there is usually long propagation delay and higher end to end time [9]. In acoustic communication bandwidth is very limited which is less than $100 \mathrm{KHz}$. In underwater scenarios, sensor nodes are usually considered static but it is also considered that they may move from 1 to 3 meter/second due to flow of water[10]. Sensor nodes used in underwater network are battery operated and it is almost impossible to replace its batteries. In underwater applications a multi-hop or multipath network is required and data is forwarded by passing all nodes towards sink. Once data is received at any of the sink then data is forwarded to concerned node through radio transmission[11]. While using those routing protocols which requires higher bandwidth, usually has higher delay at the nodes end[12]. As we know that acoustic communication does not support higher bandwidth so using routing protocols that are used in terrestrial network will not perform good due to it higher delay and high energy consumption. Using underwater network, topology does not remains the same as node moves due to flow of water[13]. In localization based protocol, geographical network information is necessary so it possess more control messages than localization free protocol, in which no prior network information is necessary. Usually ocean are vast and covers around one hundred and forty millions square miles, which is more than 70 percent of Earth total surface, Not only it has been considered to be major source of the nourishment, but with span of time its taking a good role in transportation stuffs, defence as well as adventurous purposes and natural resources presence[14]. All its importance towards humanity, it is very strange that we know a very little of about Earth water bodies. Less than ten percent of whole ocean volume is investigated, while a large amount of area has still not been explored[6]. The increase in roles of the oceans in the lives of humans, importance of these largely unexplored area has got a lot of importance. If we see, on one hand the traditional approaches for underwater monitoring have got several disadvantages while on the other side human presence is not considered 
to be feasible for underwater environment[15]. We face very unique challenges as compared to other networks. Protocols suites that are used in other networks cannot be directly applied to underwater networks. Till date, many protocols has been proposed for underwater sensor networks[16]. These are mainly divided into two types which are localization based and localization free protocols. Localization free protocols does not require any prior geographic or network information. Most of these protocols are used in underwater networks.

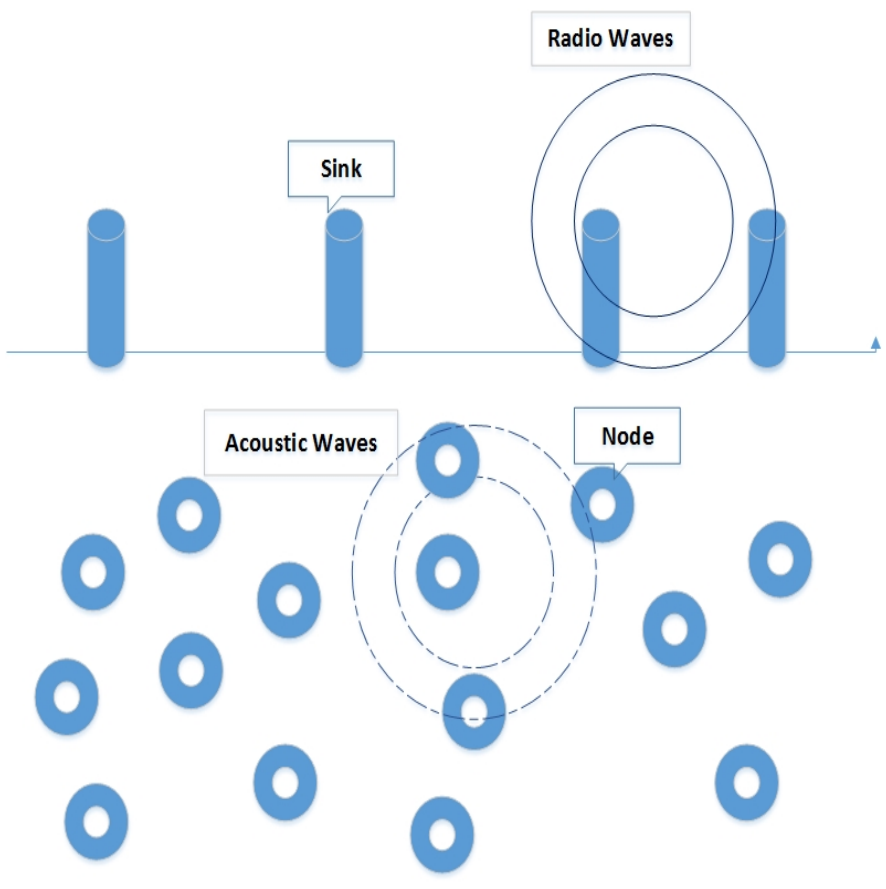

Fig. 1: UWSN Architecture

The rest of the paper is organized as follow. In section 2 related work has been discussed. In section 3 terminologies regarding routing are defined. Section 4 has discussed location free routing protocol. Evaluation and result has been discussed in section 5 and finally conclusion is drawn in section 6 .

\section{CONSTRAints In UNDERWATER Wireless SENSOR NETWORK}

UWSN carries multiple differences in comparison with terrestrial area network[17]. In which nodes are stable or move in a specified direction while in underwater networks they usually displaces their positions with the flow of water[18]. Acoustic communication is used for underwater transmission which minimizes the bandwidth for data transferring[19].

- Limited Bandwidth Acoustic channels offer very limited amount of bandwidth, as radio transmission cannot be used for underwater communication [4]. Acoustic communication requires more energy to send a small amount of data due to its lower bandwidth[20].

- Propagation Delay Due to use of acoustic communication, propagation speed becomes five times slower than that of radio frequency i.e. $1500 \mathrm{~m} / \mathrm{sec}$ [5].which obviously results in high propagation delays in the network.
- Limited Energy Nodes that are used in underwater communication are larger in size [4], hence they require larger amount of energy for communication. Furthermore, acoustic channels also required more energy for communication than terrestrial network[21],[22]. Batteries in UWSN cannot be recharged or replaced therefore use of energy efficient communication is always required to provide network with higher life time[14].

- Limited memory In UWSN nodes are small in size and therefore they have a limited amount of storage and processing capacity [6],[23].

- Variable Topology UWSN does not have a specific or static topology as flow of water make it difficult for node to remain static in one place, therefore node moves randomly[24].

\section{RELATED WORK}

In this section, relevant routing techniques in litearature are discussed. In Energy Efficient Dynamic Address Based routing (EE-DAB) [13] every node is assigned node id, s-hop id and c-hop id. Node id show the physical address of node, s-hop id consist of two digits which show how many hops away one or two sinks are. Left hop is considered as highest priority and is selected as primary route. The C-hop id also consist of 2 digits which show that how many hop the receiving nodes are away from courier nodes. acoustic communication uses more energy than that of radio communication. As wireless sensor nodes are battery operated and higher energy consumption lead towards a serious problem. Thus energy efficiency has become a major problem in underwater wireless sensor networks. In [24], a delay tolerant protocol is proposed which is called delay-tolerant data dolphin scheme. This proposed scheme is designed for delay tolerant systems and applications. In this protocols all the sensing node stay static and data sensed by static nodes are passed on to data dolphin which acts a courier nodes. So in this methodology high energy consumed hop by hop communication is avoided. Data dolphins which acts a courier nodes are provided with continuous energy. In the architecture all the static nodes are deployed in the sea bed. These static sensor goes into sleep mode if there is no data to sense and it periodically wakes up when it sense some data. After sensing some kind of desired data it simply forward this data to courier nodes which are also called data dolphins. These data dolphins take this data and deliver it to base station or sink. The number of dolphin nodes depend upon the kind of network and its application and the number of nodes deployed in the network. In [22], a virtual sink architecture is proposed where sinks are connected with each other through radio communication. In this scheme, each and every sink broadcast a hello packet which is also known as hop count update packet. After receiving hello packet by nodes, a hop count value is assigned to every sensor. These hop counts are used for selection of forwarding nodes while sending data packet from one node to another. However the proposed scheme has a few limitations which includes redundant transmission i.e. transmission of a same packet multiple times. Routing protocols which needs prior network information before send any data over the network are called localization based routing protocols. These protocol usually 
need geographical information of all node in the network as well as information about sink location. These protocols are considered to be less energy efficient most of energy is wasted in collecting their geographical information. These records are updated dynamically after fixed interval of time as nodes position may changes due to water flow. Routing protocols basically need the assumption of sensor nodes in underwater sensor networks [2]. In localization based routing protocols a node need the information of all the network nodes as well as of sink like in this scenario prior network information is needed for a node [8], [24], [21]. In [25], Focused Beam routing protocol requires geographical information of itself and as of destination. It uses Ready To Send and Clear To Send mechanism to forward data. Sender protocol transmit the RTS and receiver of the packet send back CTS. In Vector Based Forwarding [23], a source node develop a vector based routing pipe starting from sender node towards sink. Various times it is hard to find an available node in the routing pipe for data forwarding. SBR-DLP [15], also known as sector base routing, with destination location prediction is a localization based routing algorithm where node is not needed to have information of its neighbor nodes. It only need to carry its own information and pre-planned movement of sink although it decreases the flexibility of the network and it will only move around in a scheduled manner. Those routing protocols which does not require any geographical information of the network are called localization free routing protocols. These protocols perform their operation without having location information of other nodes. In these kind of routing protocols, a sensor node does not require any prior network information of other network nodes [22], [14]. Most of the localization protocols work on flooding phenomenon and are considered to have fast packet delivery ratio and low end to end delay [14], [20]. In [21], Depth base routing does not need any pre network information. It just take the depth of sensor nodes into account and forward a packet. It actually compares the depth of sending node with that of receiving node so if depth of sender node is higher than that of receiver node then it will forward the data otherwise it will ignore that node. Similarly in [11], Energy Efficient DBR, it take into account the depth information as well as residual energy of the node at the time of sending data.

\section{Node ARChitecture}

A general architecture of underwater wireless sensor node is composed of five main elements. Which are energy management unit, data sensing unit, depth measuring unit, communication unit and central processing unit [21]. As show in 2.

Processing unit is responsible for all kind of data processing which energy management unit has the responsibility to manage the remaining energy of the node and consumption of energy in run time [3]. Data sensing unit is used to sense data. It always remains active even when node is in sleep mode [26]. Communication unit is responsible for all kind of data communication whereas depth measuring unit is used for measuring depth of nodes when it is deployed in sea [9].

\section{Localization Free Routing Protocols}

In UWSN, many routing protocols had been proposed [27],[10]. Each protocol has its good and bad aspects. These

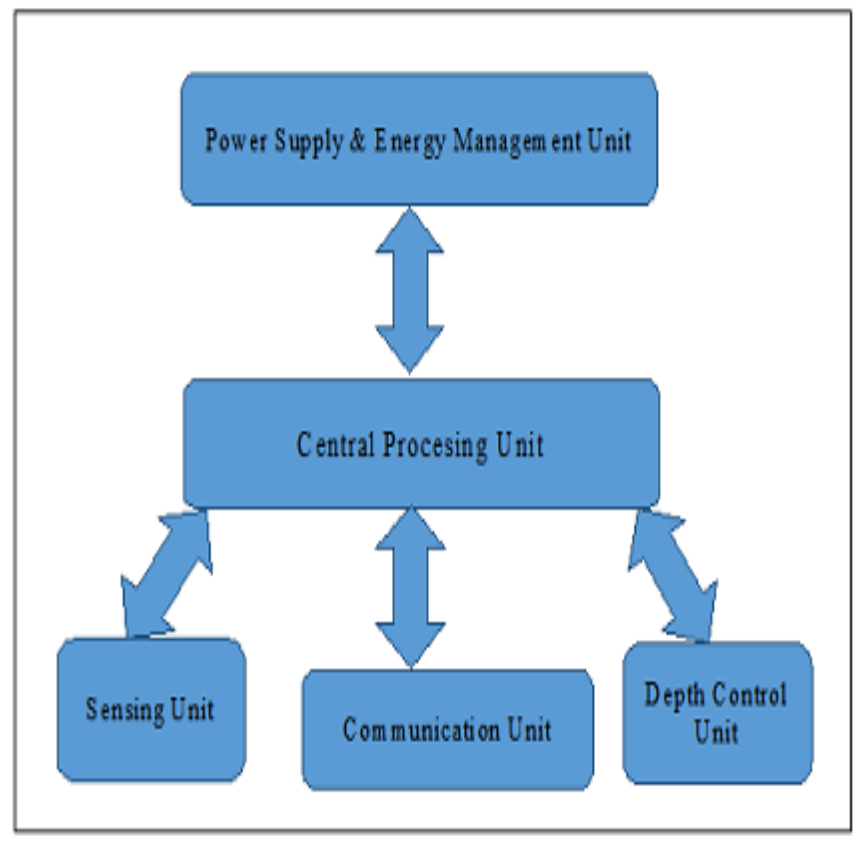

Fig. 2: Sensor Node Architecture

protocols are designed specially for underwater communication as keeping in view the limitations of the network, their low battery and low bandwidth but still there are some deficiencies that need to be addressed. Mainly routing protocols in UWSN is divided into two parts, i.e. localization based routing protocols and localization free routing protocols[8]. Localization based routing protocol comparatively requires more energy as it need prior network information[25]. Every node in the network must have detailed information of all other nodes in the network. During network initialization phase every node request other node about their current status as well as sink also broadcast ping message to know about the energy level and location of nodes. This network information is updated simultaneously after a fixed interval of time.In localization free routing protocol, it does not need any information of other nodes. This schemes consumes less energy than localization based routing protocols.

\section{A. Depth Based Routing}

Unlike localization based routing, Depth Based Routing Protocol [10] does not need any prior network information. DBR needs depth information of each node. When a node with the highest depth sense some movement, it starts sending data to higher nodes, such that it compares its depth with neighbor nodes. If send packets to only those nodes whose depth is lower than sender node. The same process continuous until packet is received by sink. This protocol is mainly concerned about depth of node. Sink are provided with continuous power. Figure 3 defines next node selection in depth based routing protocol. Where three nodes $\mathrm{n} 1, \mathrm{n} 2$ and $\mathrm{n} 3$ are in communication range of sender $\mathrm{S}$. In first step depth of receiver nodes is checked. N1 and N2 are found eligible for data forwarding as their depth is less than sender node S. Now the sender S will send the data packet to two eligible nodes N1 and N2. 


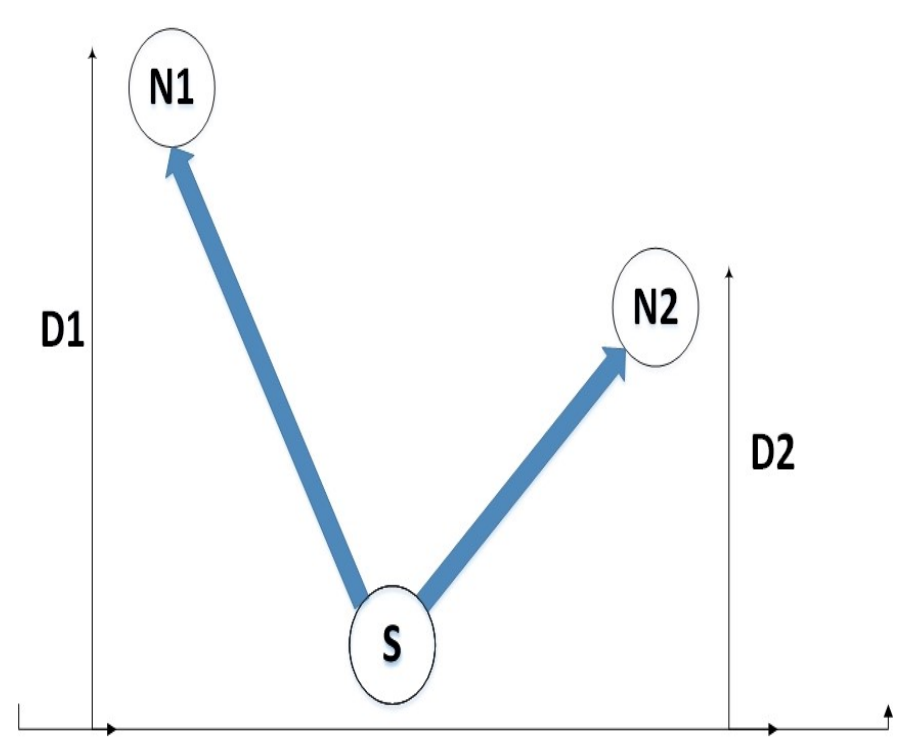

N3

Fig. 3: Depth Based Routing Protocol

The forwarding of data in broadcast manner always result in waste of energy whether a node is sending to receiving data and always leads towards low network life. DBR does not take into account any other parameter then depth, which leads towards a few drawbacks. Network life of network where DBR is used, will be less as it will always sends the data to the same higher node. Which will decrease the number of alive nodes. There is no proper mechanism for path selection in DBR as neither proper strategy is used for efficient path nor shortest path is selected.

\section{B. Hop by Hop Dynamic Addressing Based Routing}

In H2-DAB [20], dynamic addresses are assigned to nodes and destination ID is set to 0 for all nodes. No pre-network information is required in this protocol. In first step of network setup, a hop id is assigned to each node. Every node in the network will have two type of addresses, node id and hop id. Node id is physical address of node while node id changes with change in location In H2-DAB the assignment of Hop IDs which are assigned from top to bottom. Node having lower depth are assigned lower hop id, like node which is nearest will have hop id of 1 . Similarly nodes having higher depth are assigned higher hop IDs. H2- DAB supports multi sink architecture, where multiple sink are installed on shore. Those sinks are connected with each other through radio communication. Data packet received at any sink is considered received. However this approach might create problems where a node cannot find in range, any node which has lower hop id from sender node. In case of failure at finding suitable node in first attempt, sender will retransmit data packet and then wait again for specified amount of time. If results were still the same then sender node will forward data to a node having nearly or equal hop id as sender node. This process results in energy wastage.

\section{Energy Efficient Depth Based Routing Protocol}

In EE-DBR [14], protocol when a node forwards its data, it takes into account the depth of the receiver node and its residual energy. When a node forwards data it first compares the depth of the receiver node with itself, if the depth of receiver node is smaller than sender then it checks the residual energy of receiver node. Node with higher residual energy and less depth among the neighbors is selected as next hop for communication. Every node has information on depth and residual energy about their neighbors, so the node with most suitable parameter is selected for communication. EE-DBR has not defined any mechanism for multi-path communication. A node may forward data to node which is far away from sender and will results in higher energy consumption. Similarly no parameter has been taken into account to define a shortest and efficient path towards sink.

\section{Evaluation AND Results}

This paper will analyze the performance of location free routing protocols through various evaluation techniques. In this simulation three protocols i.e. DBR, EEDBR and H2-DAB are compared through simulation on the basis of network delivery ratio, path loss, network life time, number of alive nodes left and total energy consumption. Below is the parameter metrics that is taken into consideration while performing simulation.

\section{A. Simulation Parameters}

This simulation is carried out on area of $100 \mathrm{~m} \times 100 \mathrm{~m}$ with 225 node and simulation time is 9000 rounds. We have simulated DBR, EEBR and H2-DAB on the basis of total end to end delay, path loss, path loss of network packet delivery ratio and total consumed energy. Simulation results are discussed below.

TABLE I: Simulation Parameters

\begin{tabular}{|c|c|}
\hline Parameters & Value \\
\hline Network Size & $100 \mathrm{~m} \mathrm{X} \mathrm{100m}$ \\
\hline Total Nodes & 225 \\
\hline Initial Energy & $25 \mathrm{~J}$ \\
\hline Packet Size & 1024 bits \\
\hline Number of Sink & 4 \\
\hline Transmission Range & 100 meter \\
\hline Rounds & 9000 \\
\hline
\end{tabular}

\section{B. Simulation Results}

The above explained terminologies are taken into consideration during simulation while comparing DBR, EEDBR and $\mathrm{H} 2-\mathrm{DAB}$. Results gained are discussed below.

1) End To End Delay: In figure 4, three protocols are compared with respect to end to end delay. It shows that protocol which need prior network information like H2-DAB have more end to end delay while protocols like DBR and EE-DBR have low end to end delay. DBR and EEDBR uses multi-hop mechanism, when a sender node forward a packet to all available nodes in its range. Data is forwarded on multiple path simultaneously and there is no data loss even if one path fails. $\mathrm{H} 2-\mathrm{DAB}$ has higher end to end delay due to unavailability of appropriate node. 


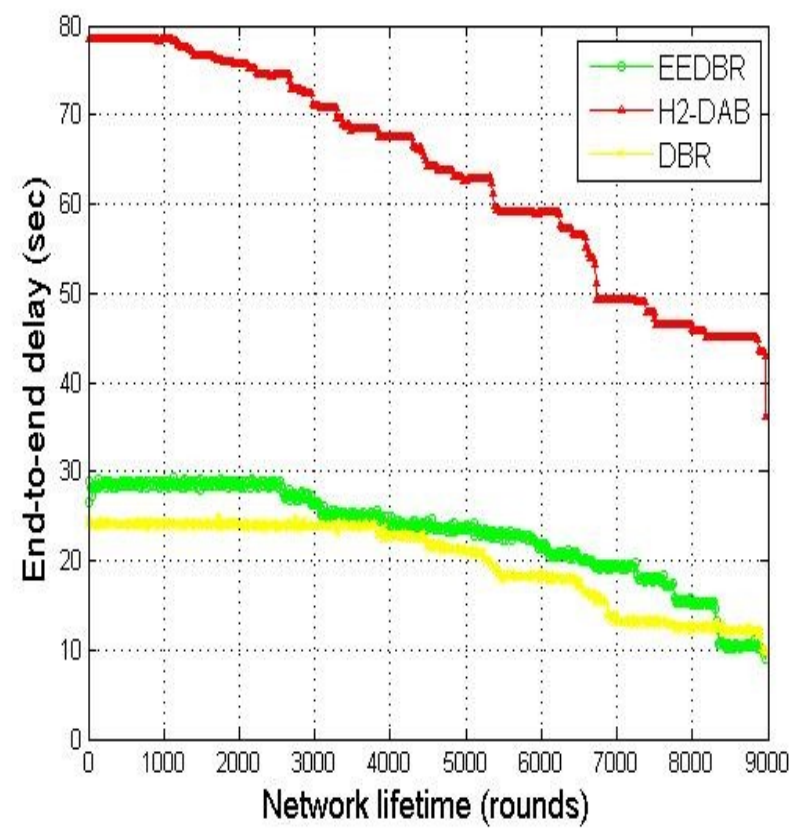

Fig. 4: End to End Delay

2) Path Loss: Figure 5, has compared path loss of network in DBR, EEDBR and H2-DAB. This figure clearly shows that path loss in H2-DAB is less while greater in DBR and EEDBR comparatively.

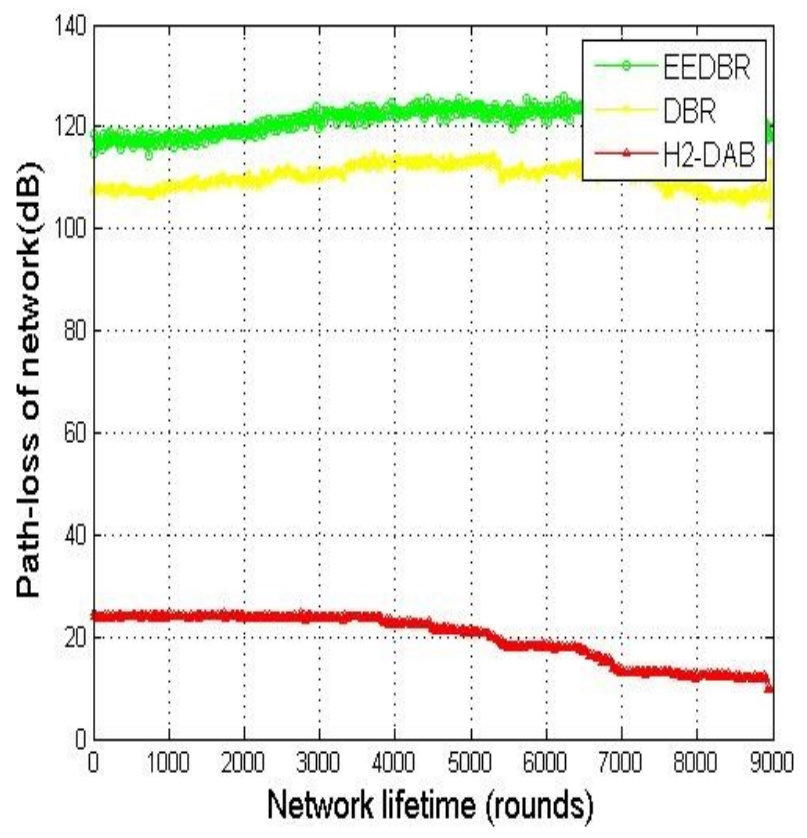

Fig. 5: Path Loss of Network

3) Packet Delivery Ratio: Figure 6 has compared packet delivery ratio in the above defined network using DBR, EEDBR and H2-DAB. The graph show delivery ratio in $\mathrm{H} 2-$
DAB is higher as compare to other two while in DBR and EE-DBR it is almost the same.

Figure 7 shows that total amount of consumed energy where DBR has consumed more energy than other two protocol because of its flooding nature. $\mathrm{H} 2-\mathrm{DAB}$ has consumed less energy of all and remained consitent throughout the process. DBR, EE-DBR and H2-DAB are compared through simulation with respect to total energy consumption, end to end delay, path loss, packet delivery ratio and number of alive nodes. The graphs show that DBR showed good results in end to end delay and packet delivery ratio while number of alive nodes are less using DBR as compared with EE-DBR and H2-DAB when compared at a certain stage. Packet delivery ratio in $\mathrm{H} 2-$ DAB is low when compared to other two. Using EE-DBR less energy is consumed when compared to other two protocols through simulation.



Fig. 6: Packet Delivery Ratio

4) Total Energy Consumption: Figure 7 shows that total amount of consumed energy where DBR has consumed more energy than other two protocol because of its flooding nature. $\mathrm{H} 2$-DAB has consumed less energy of all and remained consitent throughout the process. DBR, EE-DBR and H2-DAB are compared through simulation with respect to total energy consumption, end to end delay, path loss and packet delivery ratio. The graphs show that DBR showed good results in end to end delay and packet delivery ratio. Packet delivery ratio in $\mathrm{H} 2-\mathrm{DAB}$ is low when compared to other two routing protocols. Using EE-DBR, less energy is consumed when compared to other two protocols through simulation.

\section{CONCLUSION}

In this paper we have compared the state of the art routing protocols in UWSN. Routing in UWSN is challenging and requires energy efficient techniques. While designing any 


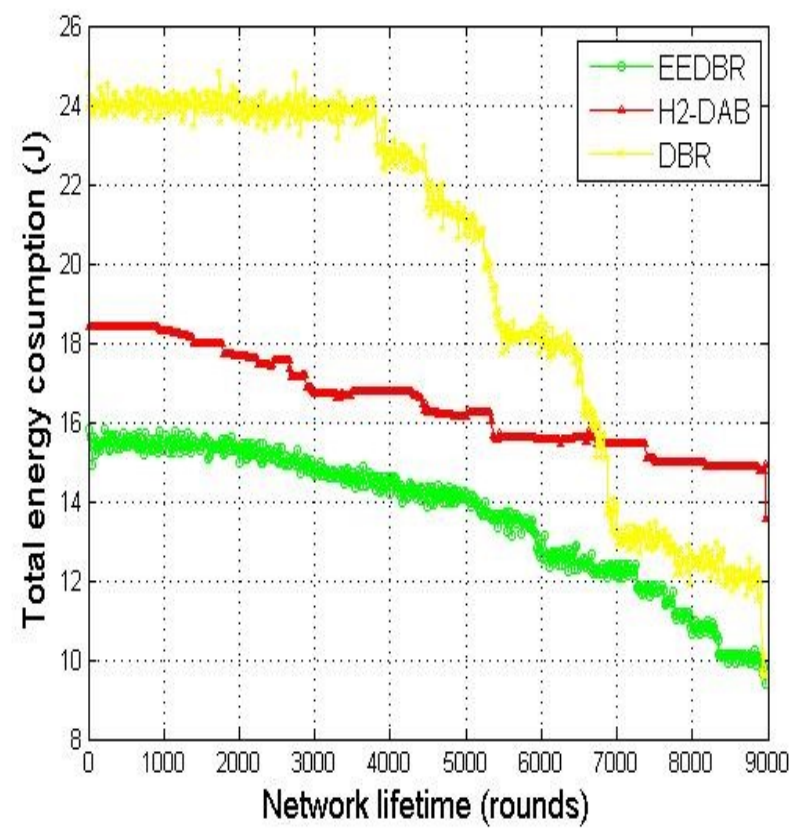

Fig. 7: Total Energy Consumption

routing protocol one should keep in view the requirement for specific application. The performance of routing protocols mostly rely on reliability, availability, energy efficiency, multi and efficient path selection, number of alive nodes and end to end delay. These all challenges attracts researchers to work in this area. Which has made UWSNs very fast growing area. Results show that H2-DAB has higher end to end delay but on the other hand it has lower network path loss than DBR and EEDBR. Packet delivery ration and network path loss of DBR and EEDBR are almost same with a little difference. There is no silver bullet for routing in UWSN and thus a lot of work could be done in the future.

\section{REFERENCES}

[1] C. Giantsis and A. A. Economides, "Comparison of routing protocols for underwater sensor networks: a survey," International Journal of Communication Networks and Distributed Systems, vol. 7, no. 3-4, pp. 192-228, 2011.

[2] N. Ilyas, M. Akbar, R. Ullah, M. Khalid, A. Arif, A. Hafeez, U. Qasim, Z. A. Khan, and N. Javaid, "Sedg: Scalable and efficient data gathering routing protocol for underwater wsns," Procedia Computer Science, vol. 52, pp. 584-591, 2015.

[3] J. L. Tangorra, S. N. Davidson, I. W. Hunter, P. G. Madden, G. V. Lauder, H. Dong, M. Bozkurttas, and R. Mittal, "The development of a biologically inspired propulsor for unmanned underwater vehicles," IEEE Journal of Oceanic Engineering, vol. 32, no. 3, pp. 533-550, 2007.

[4] I. F. Akyildiz, D. Pompili, and T. Melodia, "Challenges for efficient communication in underwater acoustic sensor networks," ACM Sigbed Review, vol. 1, no. 2, pp. 3-8, 2004.

[5] I. F. Akyildiz, D. Pompili, and Melodia, "Underwater acoustic sensor networks: research challenges," Ad hoc networks, vol. 3, no. 3, pp. 257-279, 2005

[6] J. Heidemann, W. Ye, J. Wills, A. Syed, and Y. Li, "Research challenges and applications for underwater sensor networking," in Wireless Communications and Networking Conference, 2006. WCNC 2006. IEEE, vol. 1. IEEE, 2006, pp. 228-235.
[7] N. Chirdchoo, W.-S. Soh, and K. C. Chua, "Sector-based routing with destination location prediction for underwater mobile networks," in Advanced Information Networking and Applications Workshops, 2009. WAINA'09. International Conference on. IEEE, 2009, pp. 1148-1153.

[8] E. Felemban, F. K. Shaikh, U. M. Qureshi, A. A. Sheikh, and S. B. Qaisar, "Underwater sensor network applications: A comprehensive survey," International Journal of Distributed Sensor Networks, vol. 11, no. 11, p. 896832, 2015.

[9] R. Manjula and S. S. Manvi, "Issues in underwater acoustic sensor networks," International Journal of Computer and Electrical Engineering, vol. 3, no. 1, p. 101, 2011.

[10] H. Yan, Z. J. Shi, and J.-H. Cui, "Dbr: depth-based routing for underwater sensor networks," in International conference on research in networking. Springer, 2008, pp. 72-86.

[11] A. Wahid and D. Kim, "An energy efficient localization-free routing protocol for underwater wireless sensor networks," International journal of distributed sensor networks, vol. 8, no. 4, p. 307246, 2012.

[12] M. Ayaz and A. Abdullah, "Hop-by-hop dynamic addressing based (h2-dab) routing protocol for underwater wireless sensor networks," in Information and Multimedia Technology, 2009. ICIMT'09. International Conference on. IEEE, 2009, pp. 436-441.

[13] M. Ayaz, A. Abdullah, I. Faye, and Y. Batira, "An efficient dynamic addressing based routing protocol for underwater wireless sensor networks," Computer Communications, vol. 35, no. 4, pp. 475-486, 2012.

[14] M. Ayaz and A. Abdullah, "Underwater wireless sensor networks: routing issues and future challenges," in Proceedings of the 7th International Conference on Advances in Mobile Computing and Multimedia. ACM, 2009, pp. 370-375.

[15] E. A. Carlson, P.-P. Beaujean, and E. An, "Location-aware routing protocol for underwater acoustic networks," in OCEANS 2006. IEEE, 2006, pp. 1-6.

[16] M. Patil and R. C. Biradar, "A survey on routing protocols in wireless sensor networks," in Networks (ICON), 2012 18th IEEE International Conference on. IEEE, 2012, pp. 86-91.

[17] P. Xie, J.-H. Cui, and L. Lao, "Vbf: vector-based forwarding protocol for underwater sensor networks," in International Conference on Research in Networking. Springer, 2006, pp. 1216-1221.

[18] N. Nicolaou, A. See, P. Xie, J.-H. Cui, and D. Maggiorini, "Improving the robustness of location-based routing for underwater sensor networks," in OCEANS 2007-Europe. IEEE, 2007, pp. 1-6.

[19] D. Hwang and D. Kim, "Dfr: Directional flooding-based routing protocol for underwater sensor networks," in OCEANS 2008. IEEE, 2008, pp. 1-7.

[20] M. Erol and S. Oktug, "A localization and routing framework for mobile underwater sensor networks," in INFOCOM Workshops 2008, IEEE. IEEE, 2008, pp. 1-3.

[21] K. Anupama, A. Sasidharan, and S. Vadlamani, "A location-based clustering algorithm for data gathering in $3 \mathrm{~d}$ underwater wireless sensor networks," in Telecommunications, 2008. IST 2008. International Symposium on. IEEE, 2008, pp. 343-348.

[22] N. Aitsaadi, N. Achir, K. Boussetta, and G. Pujolle, "Differentiated underwater sensor network deployment," in Oceans 2007-Europe. IEEE, 2007, pp. 1-6.

[23] J. M. Jornet, M. Stojanovic, and M. Zorzi, "Focused beam routing protocol for underwater acoustic networks," in Proceedings of the third ACM international workshop on Underwater Networks. ACM, 2008, pp. $75-82$.

[24] K. Ali and H. Hassanein, "Underwater wireless hybrid sensor networks," in Computers and Communications, 2008. ISCC 2008. IEEE Symposium on. IEEE, 2008, pp. 1166-1171.

[25] E. Magistretti, J. Kong, U. Lee, M. Gerla, P. Bellavista, and A. Corradi, "A mobile delay-tolerant approach to long-term energy-efficient underwater sensor networking," in Wireless Communications and Networking Conference, 2007. WCNC 2007. IEEE. IEEE, 2007, pp. 2866-2871.

[26] R. Manjula and S. S. Manvi, "Issues in underwater acoustic sensor networks," International Journal of Computer and Electrical Engineering, vol. 3, no. 1, p. 101, 2011.

[27] E. Felemban, F. K. Shaikh, U. M. Qureshi, A. A. Sheikh, and S. B. Qaisar, "Underwater sensor network applications: A comprehensive 
(IJACSA) International Journal of Advanced Computer Science and Applications,

Vol. 8, No. 3, 2017

survey," International Journal of Distributed Sensor Networks, vol. 11, no. 11, p. $896832,2015$. 